\title{
FINITE VOLUME ANALYSIS OF SPIRAL MOTION IN A RECTANGULAR LID-DRIVEN CAVITY
}

\author{
T. P. CHIANG, ROBERT R. HWANG AND W. H. SHEU* \\ Department of Naval Architecture and Ocean Engineering, National Taiwan University, 73 Chou-Shan Rd., Tapei, Taiwan
}

\begin{abstract}
SUMMARY
With the purpose of providing physical insight into the developing spanwise flow motion and identifying the presence of Taylor-Görtler-like vortices, we conducted a flow simulation in a rectangular cavity defined by a square cross-section and a spanwise aspect ratio of 3:1. The governing equations were solved for the transient processes by using a finite volume method in conjunction with segregated solution procedures. In the present work, attention is placed on the spiralling corner vortices near the two end walls and the longitudinal meandering Taylor-Görtler-like vortices. The investigated Reynolds number is taken to be 1500 . As a vehicle for the present flow simulation, validation against analytic data was carried out first for a configuration similar to the problem of interest. This study demonstrates the feasibility of the employed computer code.
\end{abstract}

KFY WORDS: Taylor-Görtler-like vortices; spiralling comer vortices

\section{INTRODUCTION}

Having reached a high degree of sophistication in computing power and computer graphics, together with the rapid advancement in numerical methods, the technique of computational mechanics has received increasing use within the computational fluid dynamics (CFD) community and is now applicable to large-scale flow predictions. Of particular importance is the fact that computer simulations are suitable for parametric studies. These attractive characteristics have prompted analysts to explore flow developments of physical importance. Fluid flows are by nature three-dimensional, so any prediction in a domain of two dimensions is subject to doubt. Partly owing to a lack of turbulence modelling, which is still regarded as one of the greatest stumbling blocks for scientific and technological development, and partly owing to limitations on CPU and memory for three-dimensional turbulent flow simulations, we only consider flow conditions classified as laminar.

In fluid engineering the presence of vortices has a strong association with the pressure drop and, likewise, the heat transfer. For development engineers it is thus critical to gain more physical insight into the interactive behaviour among vortices of different sizes and characters. A well-known benchmark test configured as a lid-driven cavity is best suited to manifesting this problem and, furthermore, the onset of flow unsteadiness and instability. This problem has been extensively studied for more than three decades and draws continuous attention for two reasons. First, the configuration is

* Corresponding author.

CCC 0271-2091/96/040325-22

Received May 1995

(C) 1996 by John Wiley \& Sons, Ltd. Revised November 1995 
comparatively simple for conducting experiments or analyses. Second, even though the geometry is fairly simple, the flow physics exhibited is far from simple.

Because a flow structure inside a lid-driven cavity is commonplace in many industrial flows, this problem has been the subject of many experimental endeavours. ${ }^{1-8}$ Subsequent to the experimental work of Pan and Acrivos, ${ }^{1}$ Taylor-Görtler-like (TGL) vortices were first observed in 1983 by Street and co-workers. ${ }^{3}$ Different Reynolds numbers and spanwise aspect ratios were considered in their series of experiments. ${ }^{4-7}$ According to their work, in a cavity of spanwise aspect ratio (SAR) $3: 1: 1$, eight pairs of TGL vortices were observed at $R e=3000$. With increasing Reynolds number, 11 pairs of TGL vortices were reported at $R e=6000$. In the range $6000<R e<8000$ the cavity is crowded with too many TGL vortices so that their interactions excite the unstable flow to such a degree that the flow system is more adequately classified as turbulent. While experimental work has been conducted at $R e=1000$ and 2000 , numerical results have seldom been discussed.

Numerical simulation of this problem dates back to the pioneer work of Burggraf. ${ }^{9}$ Much of the previous work has been directed towards analysis in two dimensions. More recently, three-dimensional investigations have become possible. Many of these studies have been carried out at $\operatorname{Re}=3200$ for a cavity of either $L: B: D=3: 1: 1^{10-13}$ or $1: 1: 1 .^{14-17}$ In the light of three-dimensional numerical studies, shear-driven cavity flow can be referred to as being nearly two-dimensional at very low Reynolds numbers. As the Reynolds number is increased, working variables become appreciable in the third dimension. Such a distinction is mainly attributable to the presence of two end walls. Of note is that TGL vortices were first predicted by Freitas et al. ${ }^{10}$ With increasing Reynolds number the presence of large disturbances may suddenly open the door to chaotic fluctuations and give rise to flow turbulence. Linear stability studies ${ }^{18}$ could also provide valuable information about the onset of instability. They are, however, hindered by mathematical difficulties in formulating a tractable linear stability eigenvalue problem. As a result, we only explore the consequences and causes of the formation of TGL vortices through three-dimensional numerical studies.

\section{THEORETICAL FORMULATION}

In this paper we are concerned with a set of three-dimensional Navier-Stokes equations. The dimensionless conservation equations capable of describing the motion of an incompressible fluid are representable in terms of pressure $p$ and velocity vector, $\mathbf{u}=(u, v, w)$ :

$$
\begin{gathered}
\frac{\partial u_{i}}{\partial x_{i}}=0 \\
\frac{\partial u_{i}}{\partial t}+\frac{\partial}{\partial x_{m}}\left(u_{m} u_{i}\right)=-\frac{\partial p}{\partial x_{i}}+\frac{1}{\operatorname{Re}} \frac{\partial^{2} u_{i}}{\partial x_{m} \partial x_{m}} .
\end{gathered}
$$

Hereafter the Reynolds number $\operatorname{Re}=U_{\mathrm{c}} B / v$ is defined on the basis of the cavity width $\mathrm{B}$, the kinematic fluid viscosity $v$ and the lid velocity $U_{c}=1$. While in the literature many sets of working variables are available, the primitive variable formulation employed here has gained wider acceptance because of the accessibility of legitimate boundary conditions. ${ }^{19}$

The use of a velocity-pressure formulation involves loading primitive unknowns. In both grid staggering and collocating ${ }^{20-23}$ strategies, erroneous node-to-node pressure oscillations can be well suppressed. Uncertainty as to what boundary condition should be used is the main reason for abandoning collocating grids when dealing with the pressure correction equation. On the control surface each primitive variable takes over a node to itself, whereas the pressure is surrounded by its adjacent velocity nodes. Thus the employment of staggered grids enables us to conduct a finite volume integration over each representative cell. 
Just as device disturbances obscure calibrated physics, prediction errors may inevitably cause simulation quality to deteriorate. Chief among them is non-alignment of grid lines with Cartesian coordinates. We can get around this difficulty by rewriting the basic equations in a non-orthogonal coordinate system through one-to-one curvilinear transformation. Although the use of body-fitted coordinate transformation is widespread, this procedure involves the challenging task of evaluating metric tensors. Improper approximation of them might seriously contaminate the solutions. To compensate for this imperfect cure, we prefer to conduct analyses at the Cartesian co-ordinates to ensure a precise flow investigation. In order to resolve high gradients in the boundary layer, nonuniform orthogonal meshes are thus considered.

In staggered grids the use of a higher-order upwind flux distretization scheme for non-linear terms enables us to effectively ameliorate velocity oscillations in high-Reynolds-number circumstances. The framework we adopte is that of QUICK, ${ }^{24}$ which has undergone extensive testing on diverse flow problems during the past two decades:

$$
\int_{V} \nabla \cdot(\mathbf{u} \Phi) \mathrm{d} V=\sum_{i=1}^{3}\left(u_{i}^{+} \Phi_{i}^{+}-u_{i}^{-} \Phi_{i}^{-}\right) A_{i}
$$

Here we designate $A_{i}$ as the control surface along the $i$-direction. Consider the control surface in Figure 1; the convective value $\Phi_{i}^{+}$of $\Phi$ can be approximated by

$$
\Phi_{i}^{+}=\Phi+\alpha\left(\Phi_{\mathrm{E}}-\Phi\right)+\text { Curv }
$$

where

$$
C u r v= \begin{cases}\alpha \beta \frac{\alpha-1}{\beta+1}\left[\Phi-\Phi_{\mathrm{E}}+\beta\left(\Phi_{\mathrm{EE}}-\Phi_{\mathrm{E}}\right)\right], & u_{i}^{+}<0 \\ \alpha \gamma \frac{\alpha-1}{\gamma+1}\left[\Phi_{\mathrm{E}}-\Phi+\gamma\left(\Phi_{\mathrm{W}}-\Phi\right)\right], & u_{i}^{+}>0\end{cases}
$$

and

$$
\alpha=\frac{\Delta}{\Delta_{\mathrm{E}}}, \quad \beta=\frac{\Delta_{\mathrm{E}}}{\Delta_{\mathrm{EE}}}, \quad \gamma=\frac{\Delta_{\mathrm{E}}}{\Delta_{\mathrm{W}}} .
$$

By nature, a coupled solution algorithm appears to be simple. Despite this substantial superiority in algorithmic simplicity, there is considerable computational expense associated with the demand for disc space in three-dimensional calculations. This is a consequence of solving for primitive variables by making use of the SIMPLE ${ }^{25}$ segregated approach.

\section{VALIDATION STUDY}

Prior to predicting the flow physics of interest in the rectangular cavity, it is best to conduct a validation study to confirm the analysis tool. For the purposes of validation and evaluation we consider a problem amenable to analytic solution. ${ }^{26}$ In order to be analogous to the investigated lid-driven problem, the test

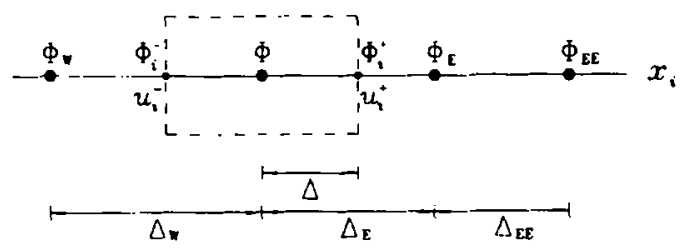

Figure 1. Grid lay-out for equations (3)-(5) corresponding to QUICK discretization scheme 


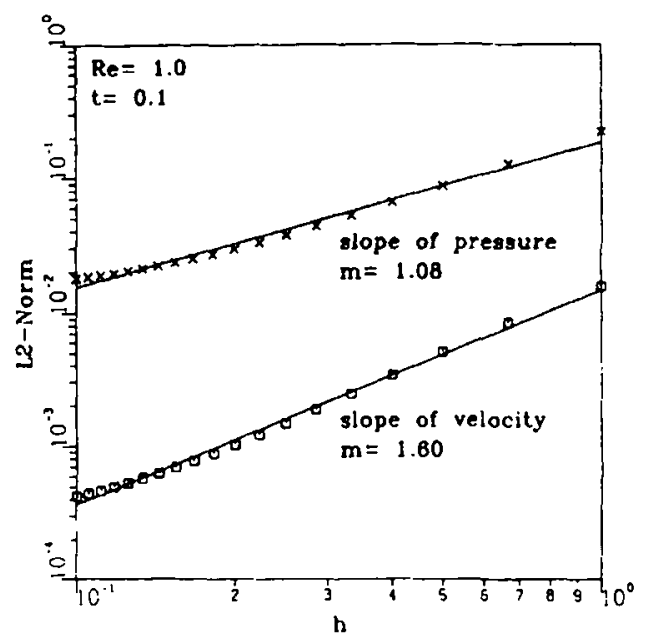

Figure 2. Rate-of-convergence test for benchmark problem defined in equations (6) and (7)

case considered is subject to the Dirichlet type of boundary conditions. On the entire boundary of the cubic cavity of length 2, prescribed nodal velocities for the case of $R e=1$ are given by

$$
\begin{aligned}
& \left.u=-a\left[\mathrm{e}^{a x} \sin (a y+d z)+\mathrm{e}^{a z} \cos (a x+d y)\right)\right] \mathrm{e}^{-d^{2} t}, \\
& \left.v=-a\left[\mathrm{e}^{a y} \sin (a z+d x)+\mathrm{e}^{a x} \cos (a y+d z)\right)\right] \mathrm{e}^{-d^{2} t}, \\
& \left.w=-a\left[\mathrm{e}^{a z} \sin (a x+d y)+\mathrm{e}^{a y} \cos (a z+d x)\right)\right] \mathrm{e}^{-d^{2} t} .
\end{aligned}
$$

In the cubic cavity $-1 \leqslant x, y, z \leqslant 1$ the exact pressure for this problem takes the form

$$
\begin{aligned}
p= & -\frac{a^{2}}{2}\left[\mathrm{e}^{2 a x}+\mathrm{e}^{2 a y}+\mathrm{e}^{2 a z}+2 \sin (a x+d y) \cos (a z+d x) \mathrm{e}^{a(y+z)}\right. \\
& \left.+2 \sin (a y+d z) \cos (a x+d y) \mathrm{e}^{a(z+x)}+2 \sin (a z+d x) \cos (a y+d z) \mathrm{e}^{a(x+y)}\right] \mathrm{e}^{-2 d^{2} t},
\end{aligned}
$$

where $a=\pi / 4$ and $d=\pi / 2$. In order to assess the solution quality, we measure prediction errors at $t=0.1$ for each primitive variable. On the basis of $n$ nodal points in total we calculate the $L_{2}$ error norms

$$
\begin{aligned}
& \varepsilon_{\mathbf{u}} \equiv\left\|\mathbf{u}-\mathbf{u}_{\mathrm{exact}}\right\|_{L_{2}}, \\
& \varepsilon_{p} \equiv\left\|p-p_{\text {exact }}\right\|_{L_{2}},
\end{aligned}
$$

where

$$
\|\Phi\|_{L_{2}}=\left(\frac{\sum_{i=1}^{n}\left(\Phi_{i}\right)^{2}}{n}\right)^{1 / 2} .
$$

Solutions are sought at constant time increment $\Delta t=1 / 160$ and different uniform grids $h=2 / 2,2 / 3, \ldots, 2 / 19,2 / 20$. We have plotted the resulting errors logarithmically against the grid spacings in Figure 2, from which we can obtain the rate of convergence for the underlying QUICK- 


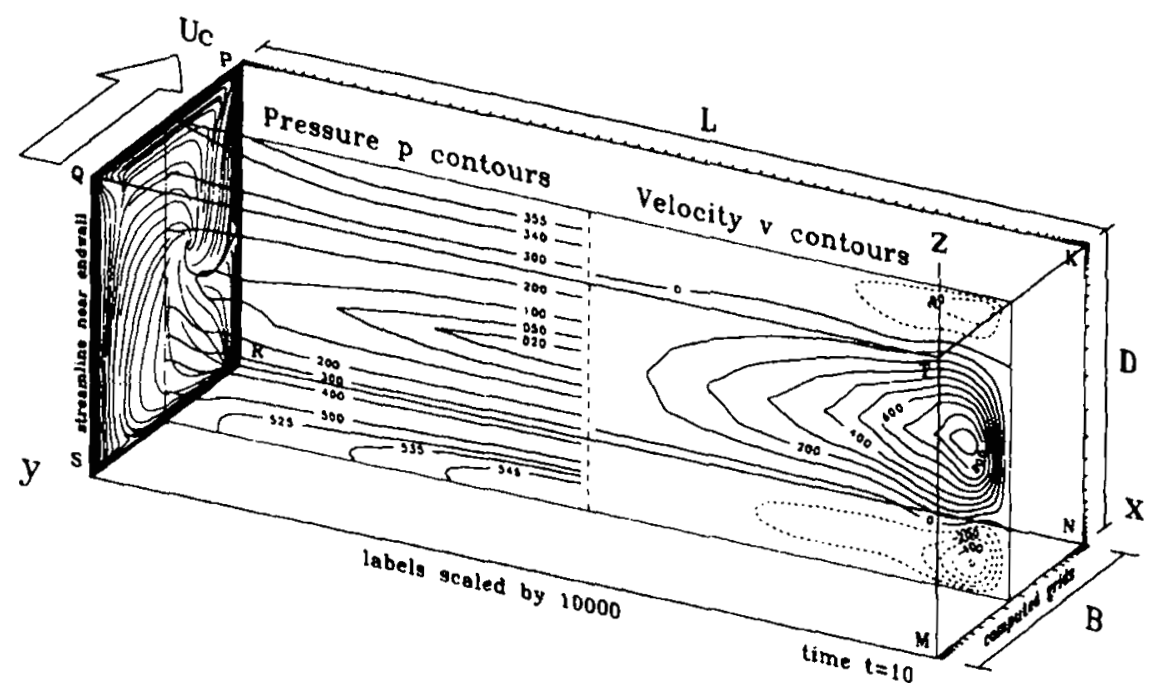

Figure 3. Description of investigated cavity of $S A R=3: 1$. Pressure contours and spanwise velocity contours are also plotted for illustration

type discretization scheme. As seen in Figure 2, close agreement is demonstrated between the calculations and the analytic data. This confirmation study supports the conclusion that the present finite volume approach can be applied to prediction of the flow physics inside a shear-driven cavity.

\section{COMPUTED RESULTS AND DISCUSSION}

\section{Problem description}

The test problem was that of the lid-driven cavity shown in Figure 3. The investigated cavity was defined by $L: B: D=3: 1: 1$, where $L$ stands for the span, $B$ the width and $D$ the height. Within the whole rectangular cavity, primitive variables were sought on a non-uniformly discretized domain of grid resolution $34 \times 91 \times 34$. For simplicity we designate PQSR and KTMN as the end walls, PKNR the downstream side wall, QTMS the upstream side wall, SMNR the lower horizontal wall and QTKP the lid plane.

The Reynolds numbers considered were 250,500,750,1000, 1250, 1500 and 2000. Regarding this series of calculations, it is worthwhile to explain why we focused our attention on $R e=1500$. The rationale can be briefly described as follows. Prior to $R e=250$ the fluid flow will most likely display a two-dimensional character. In the range $R e=500-1000$ the flow gradually develops into a smallamplitude three-dimensional state and the symmetry of the flow is still well maintained. At $R e=1250$ the flow field starts to show travelling waves that are too mild to excite TGL vortices. Suffice it to say that when $R e>1000$, transient analysis needs to account for the flow unsteadiness. The quest for the critical Reynolds number that gives a hint of the onset of TGL vortices is thus of importance. Numerical exercises for $R e=1375$ reveal that five sets of vortices taking a mushroom-like form appear over the entire span. This mobile and unsteady system is still too regular to be classified as turbulent, in the sense that the transport process undergoes periodic variation. The periodicity is estimated to be 72 . As the Reynolds number continues to increase to $R e=2000$, this periodicity-preserving behaviour disappears in the rectangular cavity filled with seven pairs of TGL vortices. The symmetric character is no longer a definitive outcome. Numerical simulation thus needs to be carried out in the entire cavity 
under these circumstances. Because we are mainly concerned with the growth of disturbances into Taylor-Görtler vortices, the investigated Reynolds number should not be so high as to yield random data that will only complicate matters. To give us a clear picture of the formation of TGL vortices, we consider a moderate Reynolds number of 1500 .

We performed the transient simulation over the entire evolution and terminated at $t=444$. Grid nodes were appropriately arranged so that physically plausible solutions were obtained. The disc space used for a marching time step was 1.8 Mbytes, while the memory taken was estimated to be 6 Mbytes. On an HP 730 each computation took approximately 10 days of CPU time.

\section{Evolution of spanwise flow motion}

Owing to the fluid viscosity, the moving lid drags the fluid flow adjacent to the upper plate and gives rise to a global flow motion. Exclusive of the interior primary recirculating cell, two less apparent secondary eddies, namely the downstream secondary eddy (DSE) and the upstream secondary eddy (USE), emerge.

According to Figure 3, decelerating fluids at the two end walls induce a favourable pressure gradient. It is this force that engulfs particles to the symmetric plane. A manifestation of the distinction between two- and three-dimensional flows is the presence of spanwise velocity. In these circumstances a threedimensional analysis becomes indispensible. Moreover, the fact that the pressure gradient changes sign at planes parallel to the symmetric plane explains why fluid particles approach the two end walls. This is conceptually identical with placing a suction pump in the core region so that fluid particles, exclusive of the primary vortex, proceed with an outward-running motion. According to Figure 3 , such a flow structure is clearly manifested by the plotted streamlines at a plane that is fairly close to the left end wall. This sheds light on why fluid particles inside two different secondary eddies are entrained to the primary core and move towards the symmetric plane.

Spanwise velocities deserve extensive consideration because they play an essential role in forming a spiral flow structure. Along with the primary circulation, the flow pattern is doomed to be spiral in the presence of appreciable spanwise velocity. In illustration of the presence of helixes, we have plotted massless particle tracers that were initially released at three representative locations in Figure 4. To begin with, we considered a fluid particle at $(0.5,1.9,0.03)$. As seen from the perspective view in Figure 4(a), a particle designated by ' $a$ ' migrates towards the upstream wall and then deflects as a result of being affected by the combined spanwise and primary flow motions. A large-radius loop of a spanwise sprialling particle track is revealed near the end wall PRSQ. In the course of this journey, particle ' $a$ ' takes $25 \%$ of the entire time to advance to location ' $b$ ' and is then quickly drawn into the primary circulation in a much smaller loop. This inward spanwise motion takes about $10 \%$ of the whole journey to move to location ' $c$ ', which is fairly close to the symmetric plane. In the ensuing sequence the spiralling particle ' $a$ ' continues to move towards the symmetric plane at an increasingly slower pace but in a larger loop. It takes approximately $60 \%$ of the entire time before marker ' $a$ ' is drawn out of the primary core and engulfed into the DSE. This is comparatively fast, as only $5 \%$ of the time is needed for a particle to travel from ' $d$ ' to ' $e$ '. Noticeable in this figure is the monotonic spriralling motion. Fluid flows in the vicinity of the DSE and USE are also described in Figure 4(b). After being released from $(0.95,1.65,0.05)$ and $(0.188,1.567,0.0708)$, particles move towards the end walls and their spanwise translation eventually stops, followed by a sudden lift to the core of the cavity. Afterwards they proceed again to the symmetry plane in a spiralling fashion. Figure 4(b) reveals that fluid particles released from the USE go forward with a smaller radius of helix in the core and can be characterized as possessing a monotonic motion. For completeness we considered the particle track that is affected by the TGL vortices. In an attempt to illuminate such flow characteristics, we considered the same particle ' $a$ ' as considered in Figure 4(a). Within a narrow span, as seen in 

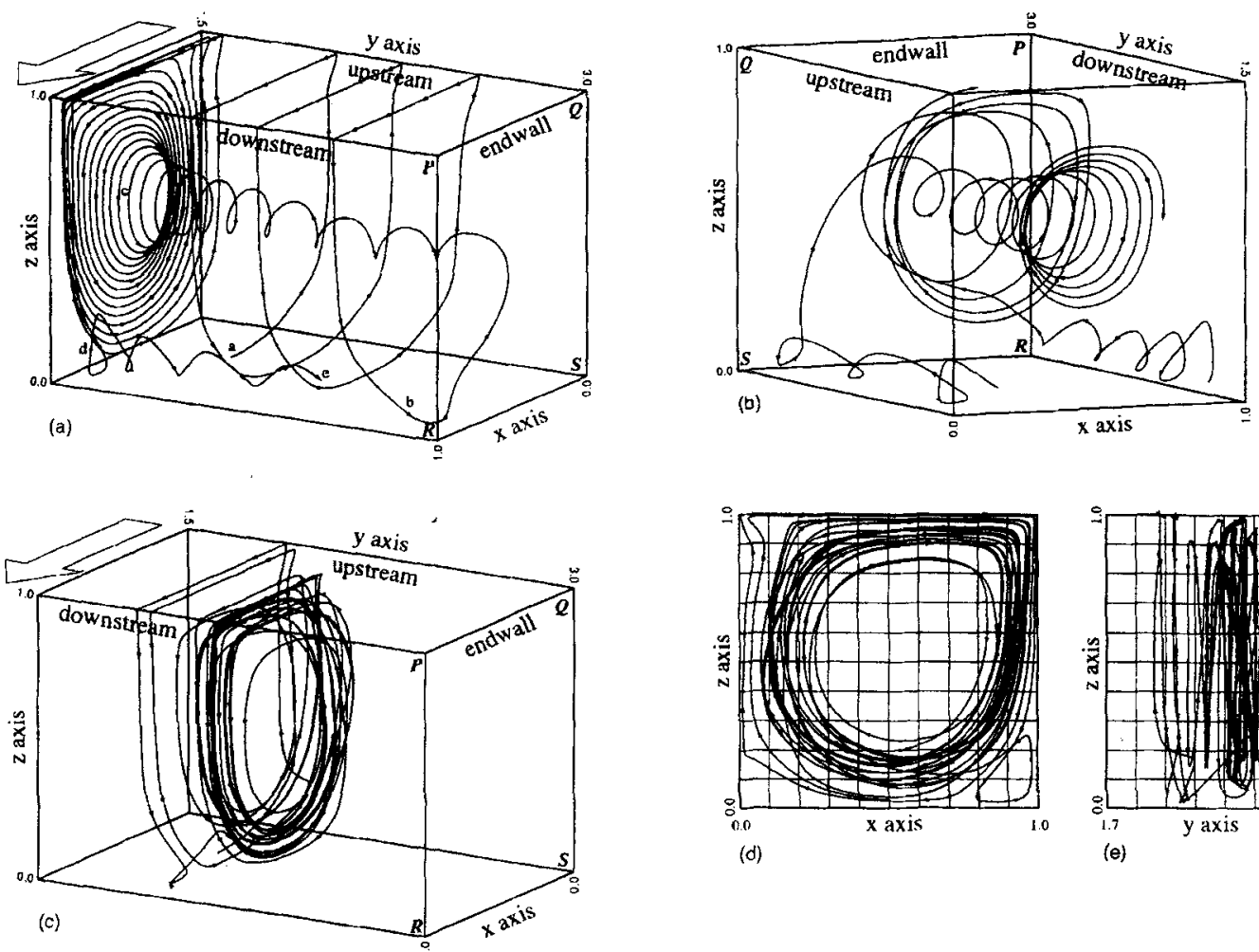

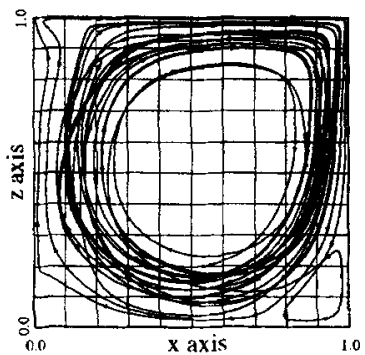

(d)

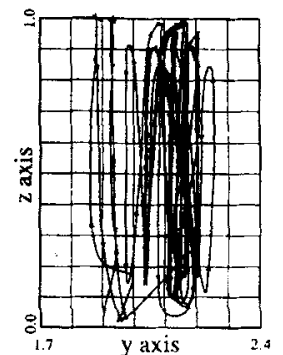

(e)

Figure 4. Illustration of spiralling particles: (a) monotonic particle track (at $t=25$ ), released from $(0.5,1 \cdot 9,0.03$ ), which has not been affected by TGL vortices; (b) particle track at $(t=25)$, released from $(0.188,1.567,0.0708)$, inside USE and particle track, released from $(0.95,1.65,0.05)$, inside DSE; (c) back-and-forth particle tracks at $(t=85)$ which have been affected by TGL vortices: (d) plots (c) viewed from $x-z$ plane; (e) plot (c) viewed from $y-z$ plane

Figures 4(c)-4(e), back-and-forth spanwise motions are visible from different views. Comparatively speaking, particle 'a' meanders in a more irregular manner when it comes across the free shear vortex, as shown in Figure $11(t=85)$. This explains why the rod-like isosurface of $v=0$ is attached to the side walls in Figure $5(\mathrm{~h})$.

The main purpose of depicting the flow structure in Figure 5 is to illustrate the variation of the flow in the spanwise direction. The impact on the global flow is manifested by the presence of the isosurface of $v=0$. Later on we will discuss the distinct characters of the isosurface of $v=0$ starting from $t=15$ and ending at $t=85$. Further evidence regarding the gradual development of the spanwise motion can be revealed from different viewpoints. In the very first stage of the lid motion the pressure established takes on a radial form at planes spanned by both longitudinal and transverse co-ordinates. As seen in Figure 6(a), the location at which the lid plane interesects with the downstream vertical plane serves as a singularity point from which a radial type of pressure distribution propagates to the core of the cavity. In the very early part of flow development the presence of the vertical end walls, along which a slowly developing boundary layer becomes visible, affects the curved primary core flow. As a result, fluid particles adjust themselves by setting up an elongated radial type of pressure distribution, as seen in Figure 6(b), to allow and support the fluid particle moving spirally towards the symmetry plane. At a later time, $t=10$, the ring-shaped pressure contour present in the inner domain shifts towards the end wall, so that this pressure becomes more flattened even in the vicinity of the symmetry plane. As time 

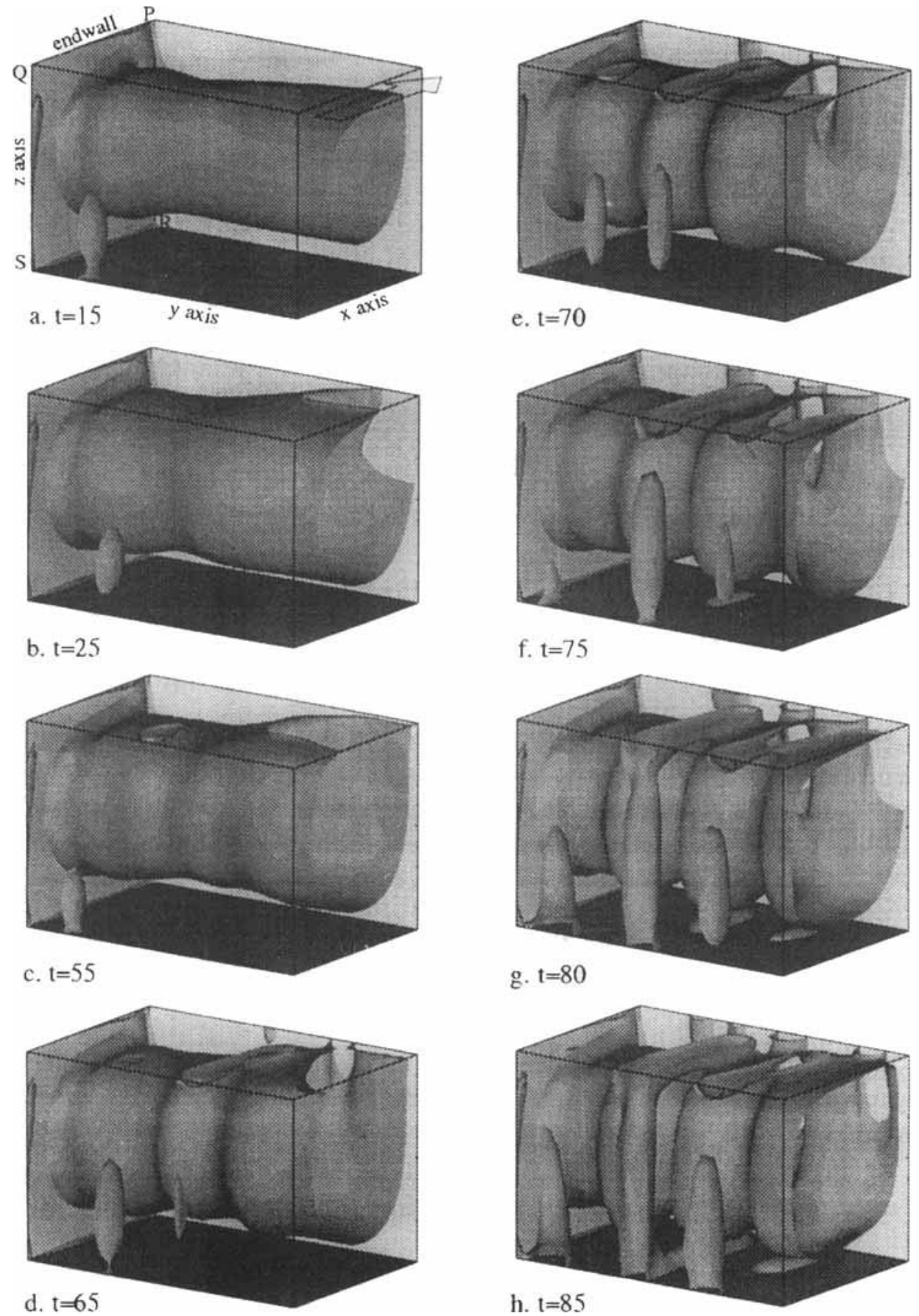

Figure 5. Contour surface of $z=0$ inside half-cavity: (a) $t=15 ;$ (b) $t:=25 ;$ (c) $t=55 ;$ (d) $t=65 ;($ c) $t=70 ;(f) t=75 ;$ (g) $t=80$; (h) $t=85$ 


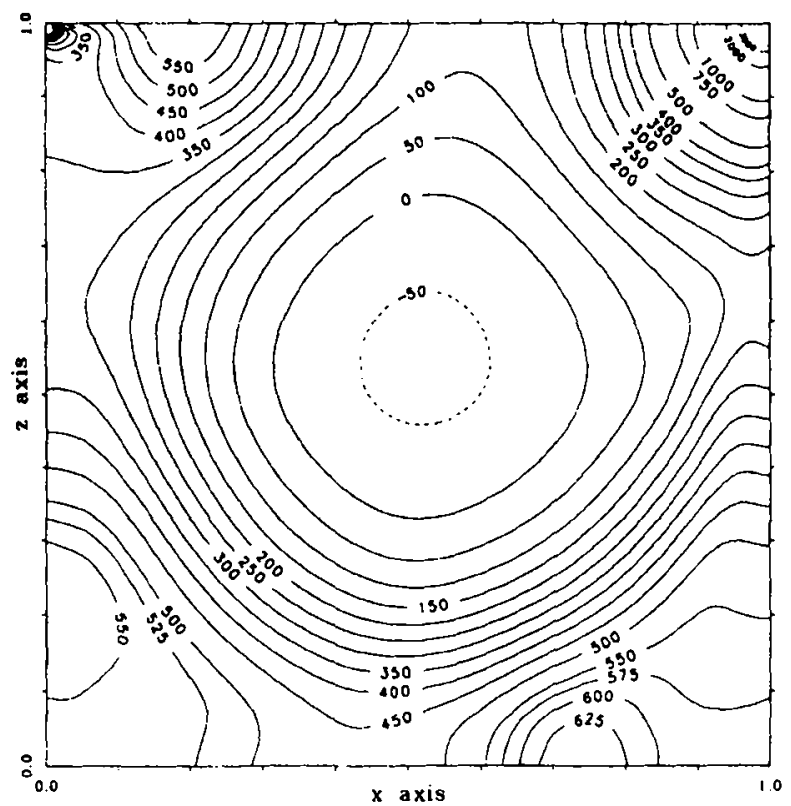

(a)
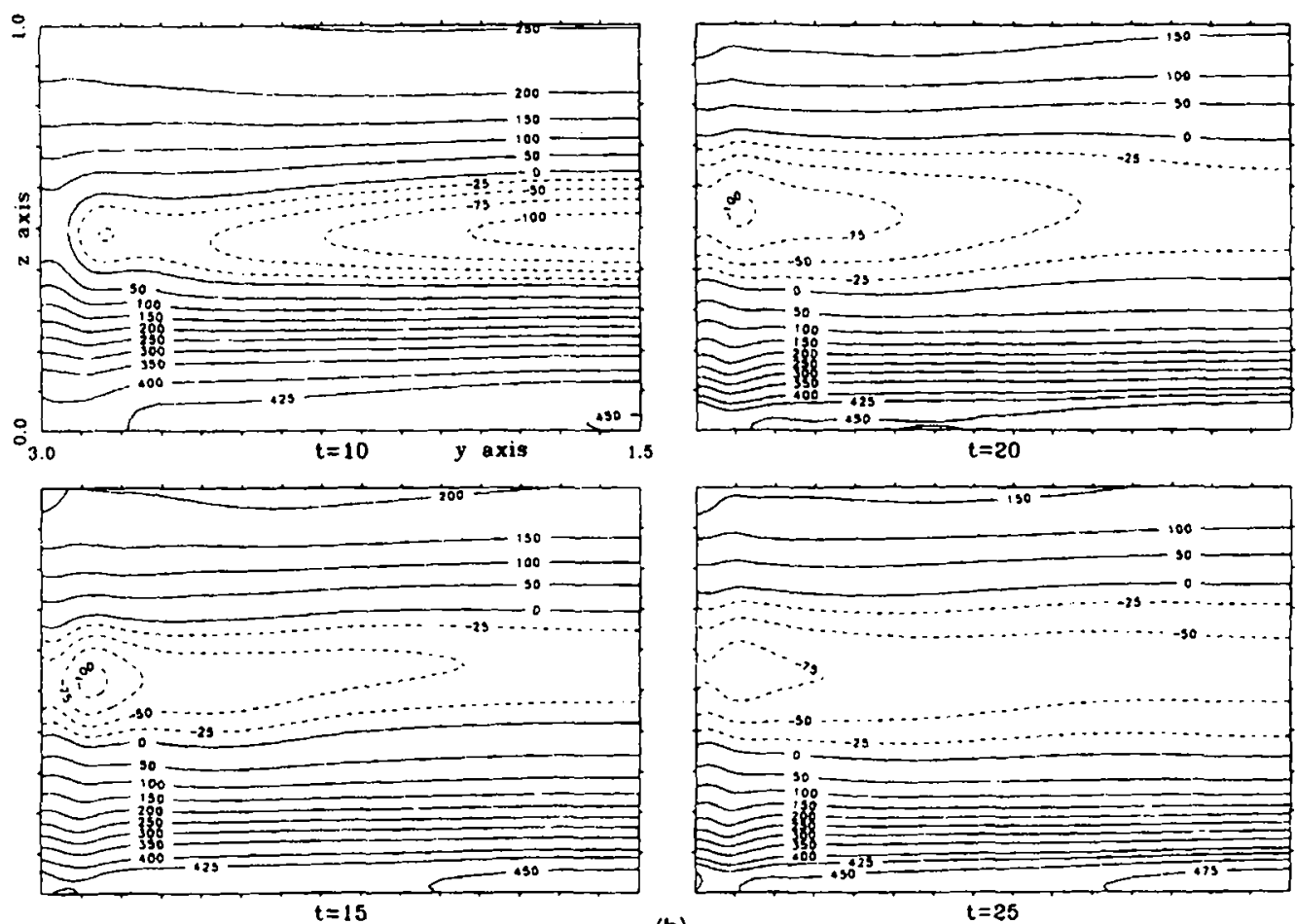

(b)

Figure 6. Computed pressure contours scaled by $10^{4}:$ (a) at $y=1.5$ plane and $t=25$; (b) at $x=0.525$ plane where $t=10,15,20$, 25 have been' considered 

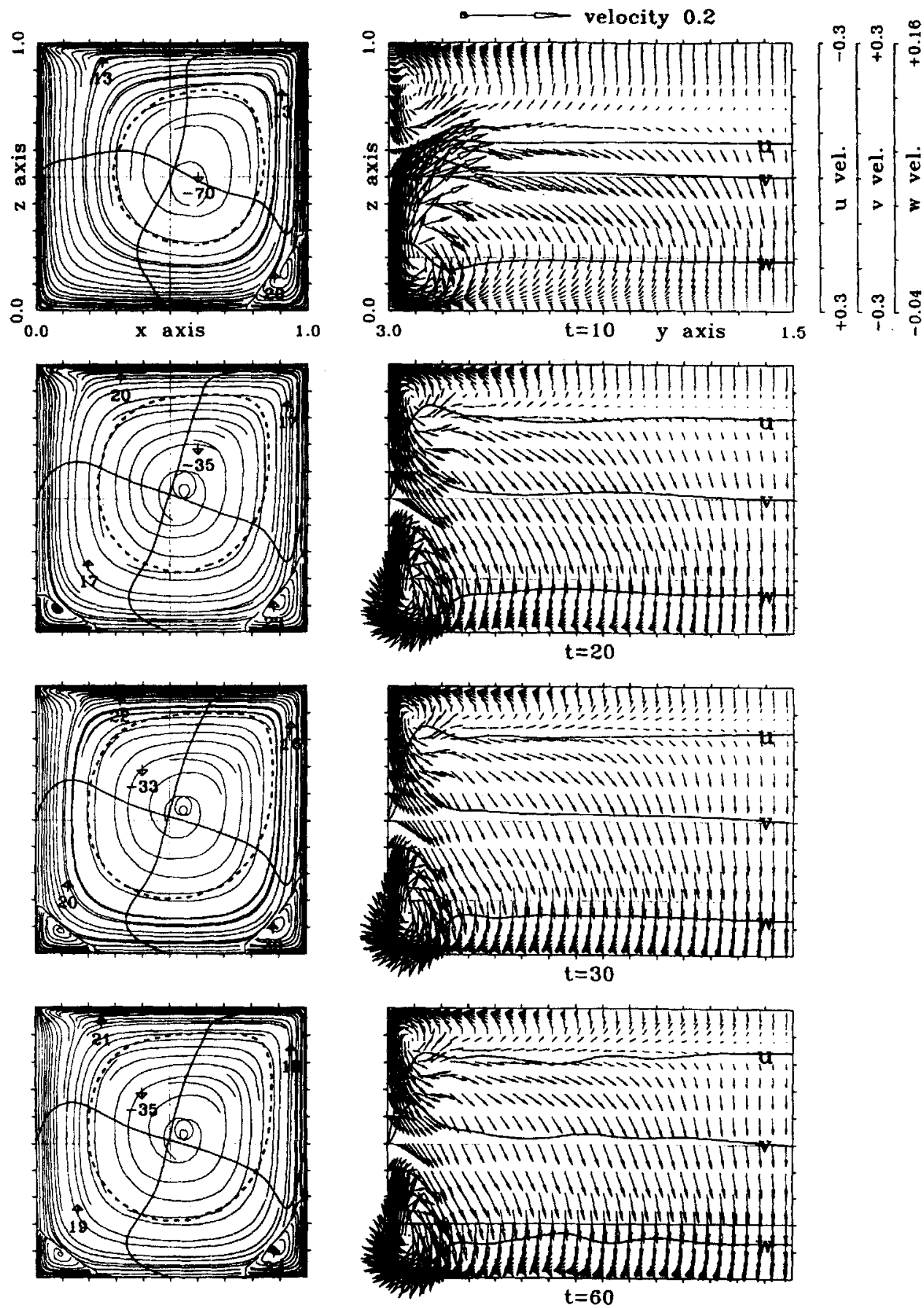

Figure 7. Evolution of flow field from $t=10$ to 85 depicted at $y=2.5$ plane and $x=0.6$ plane. The broken line on the left part stands for $v=0$ and $\uparrow / \downarrow$ are the local $\mathrm{max} / \mathrm{min}$ (scaled by $10^{3}$ ). On the right part of the plots, profiles of $u, v, w$ are plotted at $(x=0.6, y, z=0.04)$ 

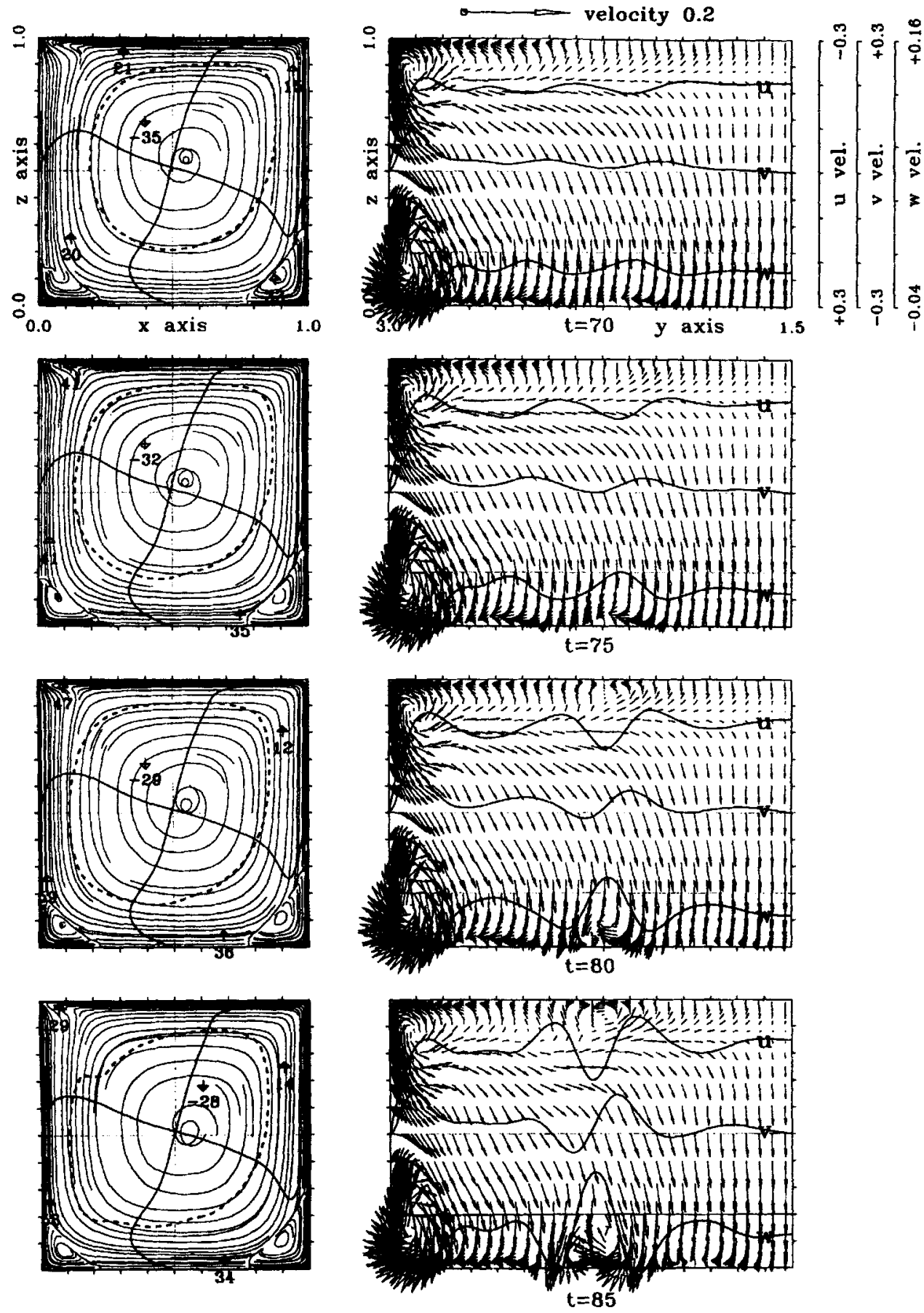

Figure 7. (continued) 
advances to $t=20$, these parallel pressure contours vary in the transverse direction. This leads to an accompanying spiral particle motion in regions adjacent to the lid plane and the bottom wall. More apparent is the radial distribution of pressure contours near the bottom wall. At this stage it can be concluded that the presence of the spanwise variation is not solely attributable to end wall effects. Instead, it is the complex combination of the spanwise pressure gradient, radial pressure gradient, centrifugal force and no-slip boundary velocity that leads to the formation of spanwise velocities.

As mentioned earlier, fluid flows in a rectangular box can be characterized as possessing eddies of distinct sizes. Because the physics of flow in this cavity is anything but simple, it is instructive to plot the prediction results in a three-dimensional context from which complex recirculating flows at different times can be clearly visualized. To arrive at this objective, we have plotted different perspective views at planes $y=2.5$ and $x=0.6$. The right part of Figure 7 reveals that the flow pattern develops gradually prior to $t=30$. Exclusive of a small perturbation in regions adjacent to the bottom wall, the change in the flow structure is, as a whole, indistinguishable between $t=30$ and 60 . At a spatial location which is fairly close to the bottom wall, say $z=0.04$, the velocity distributions of $u, v$ and $w$ at the $x=0.6$ plane vary along the spanwise direction. As seen in Figure 7, wavy velocities become clearly visible at time $t=60$. Plausible reasons leading to the wavy profiles of $u$ and $v$ by different possible destabilizing means will be discussed later on.

We now turn to the flow visualization from the perspective view at the $y=2.5$ plane. Besides streamlines, as seen in Figure 7, we have also depicted contour lines of $v=0$, the local extreme value of $v$ and the velocity distributions of $u$ and $w$ at $x=0.5$ and $z=0.5$. These plots enable us to gain physical insight into the spanwise flow motion. Presumably hindered by an increasingly developed boundary layer along the vertical end wall, together with the presence of stronger comer vortices, the local maximum velocity inside the $v=0$ tube drops dramatically in the early flow development from $t=10$ to 20 . Accordingly, the domain having the negative spanwise velocity is enlarged so that mass conservation is retained.

Prior to $t=60$ the $v=0$ contour has good alignment with the streamline. This explains why flow particles proceed in a monotonically spiralling manner. Beyond $t=70$, streamlines go against the contour surface of $v=0$. This discrepancy is pronounced, particulary at the upstream side, owing to the observed wavy contour of $v=0$ herein. In view of the degree of alignment between the $v=0$ contours and the streamlines, we may conclude that the flow structure at the downstream side is more stable than that at the upstream side for the present investigated Reynolds number.

\section{Formation of $T G L$ vortices}

To begin with, we first review some commonly encountered instabilities which tell us more about longitudinal vortices in the lid-driven cavity. As early as the 1920 s, longitudinal vortices inside the boundary layer of a heated horizontal wall were known to scientists. Later, in 1940, a similar flow structure was observed in the attached boundary layer over a concave wall. ${ }^{27}$ Görtler attributed this type of instability to the laminar flow against small disturbances in the form of longitudinal vortices. In fact, the buoyancy force in the thermally stratified layer plays a role that is analogous to the centrifubal force that gives rise to physical instabilities in the concavely curved laminar boundary layer. In between two concentric cylinders the presence of the so-called Taylor vortex instability demonstrates the consequences of the centrifugal effect. Besides this destabilizing mechanism, appreciable curved streamlines in the viscous flow system can also support Tollmien-Schlichting travelling waves. Indeed, many industrial flows allow for the coexistence of Taylor-Görtler vortices and Tollmien-Schlichting waves in addition to Rayleigh instabilities. ${ }^{28}$ Very often, interactions among these instabilities can lead to premature transition to turbulence and aid the transfer processes. 

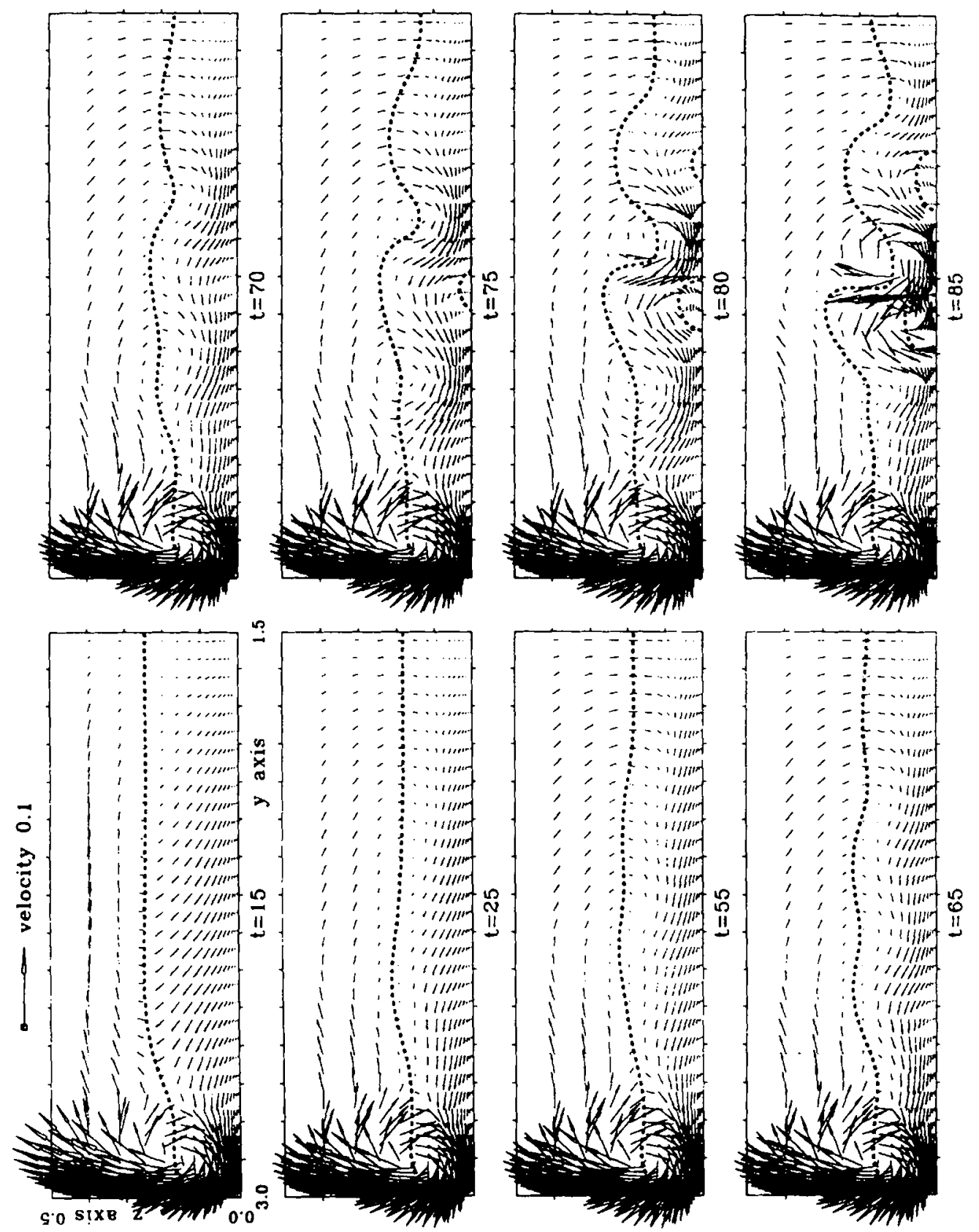

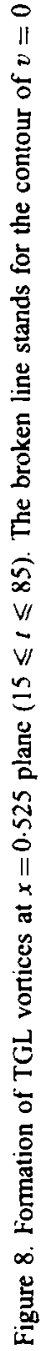



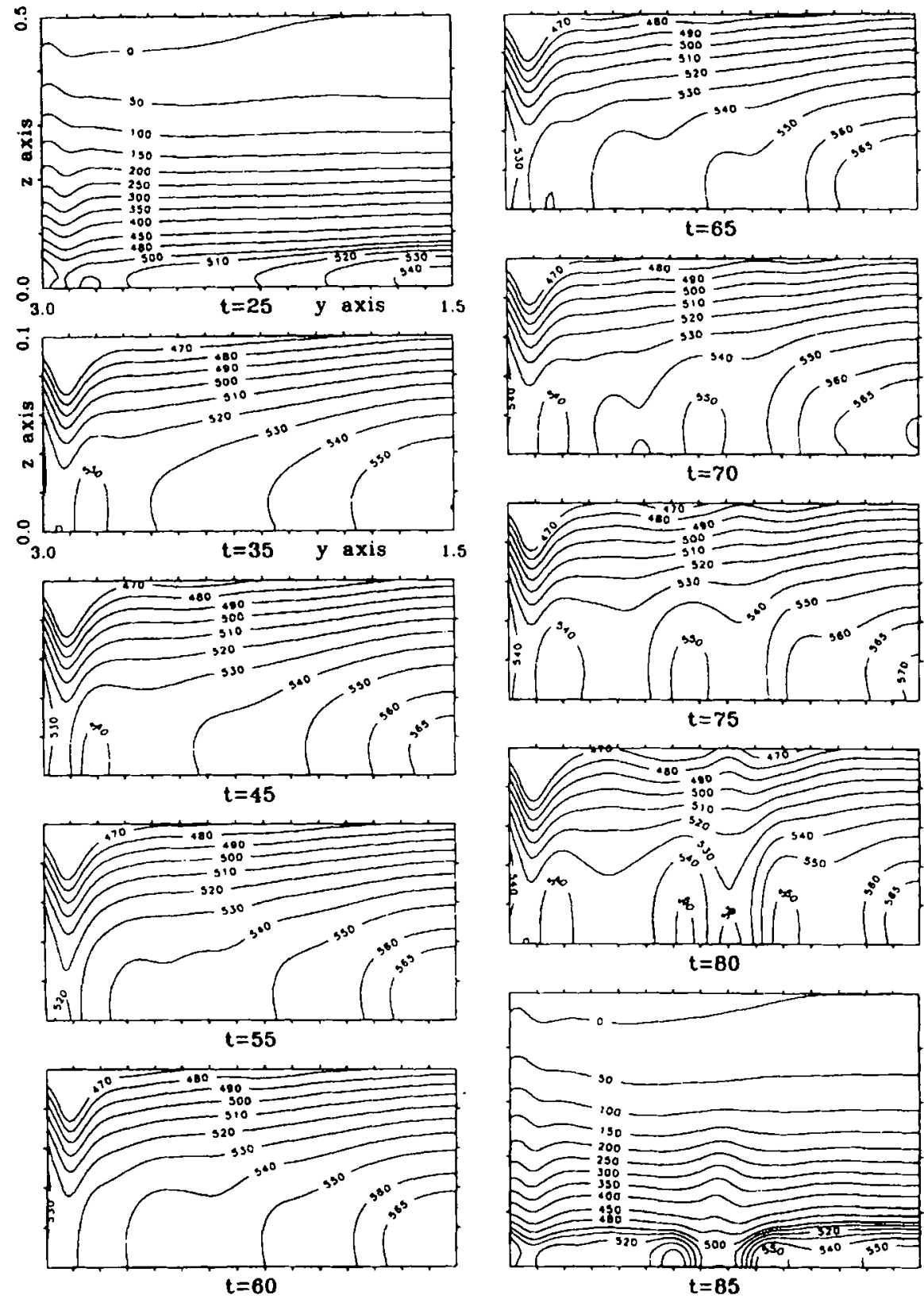

Figure 9. Pressure contours computed (scaled by $\left.10^{4}\right)$ at $x=0.525$ plane $(25 \leqslant t \leqslant 85)$

In order to give a detailed description of the evolution of TGL vortices, we have recorded the time history of velocity vectors at the $x=0.6$ plane in Figure 7 and the $x=0.525$ plane in Figure 8. From these figures we believe that as early as $t=15$ the flow is little disturbed even though the comer vortex is clearly visible. Over the half-span the boundary layer along the bottom wall is not under the influence of increasing pressure emanating from the corner region. Noticeable is the formation of a small-scale circulation zone located halfway between the symmetry plane and the end wall. As time 

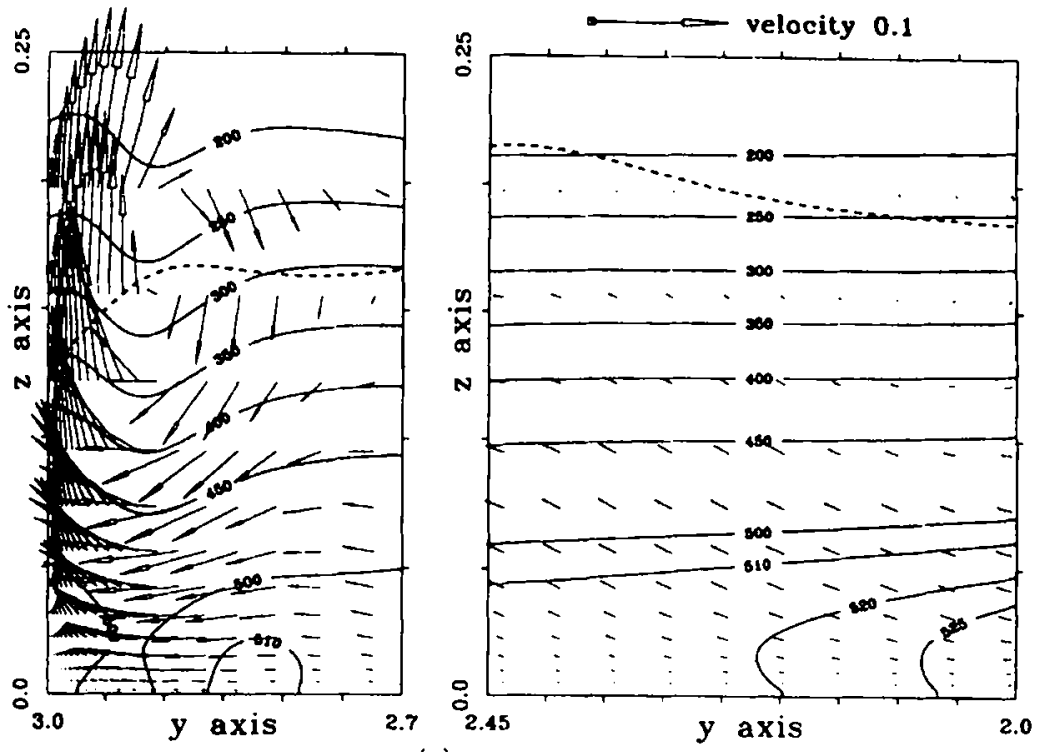

(a)
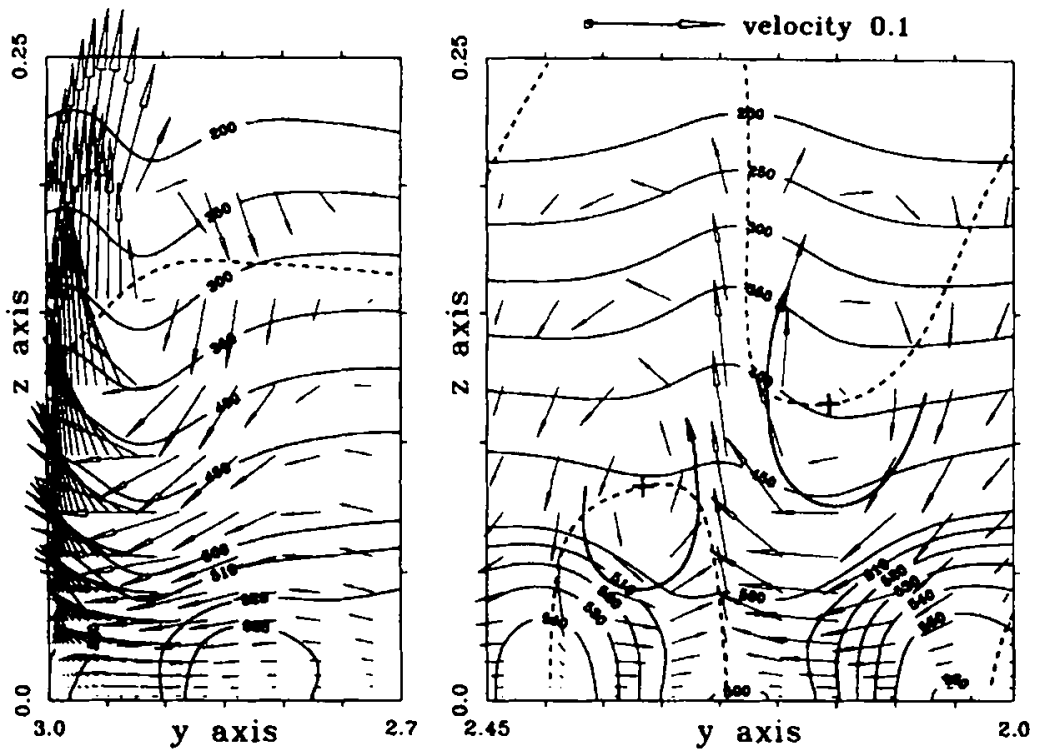

(b)

Figure 10. Illustration of corner/TGL vortices at $x=0.525$ plane: (a) at $t=25$ which is free of TGL vortices; (b) at $t=85$ where TGL vortices have been established. Contours of pressure have been scaled by $10^{4}$ and the broken line stands for $v=0$. ' + ' represents the vortex centre 
goes by, the territory of spiral particles moving towards the symmetry plane of the cavity is enlarged, as illustrated by the broken line of the contour of $v=0$ in Figure 8. At $t=70$ the contour line of $v=0$ penetrates into the end wall running spiral region. The slowly developing flow patterns cut across the isoline of $v=0$ and result in a larger and clearer circulation cell. Thus a higher rate of mixing among fluid particles can be expected in the core as well as near the bottom wall of the cavity. The isosurface of $v=0$ is continuously modified by the pressure propagation stemming radially from the centre of the corner vortex as well as from the intersection point between the symmetry plane and the bottom wall, as seen in Figure 9. In the course of flow development the opposing radial pressure modifies the
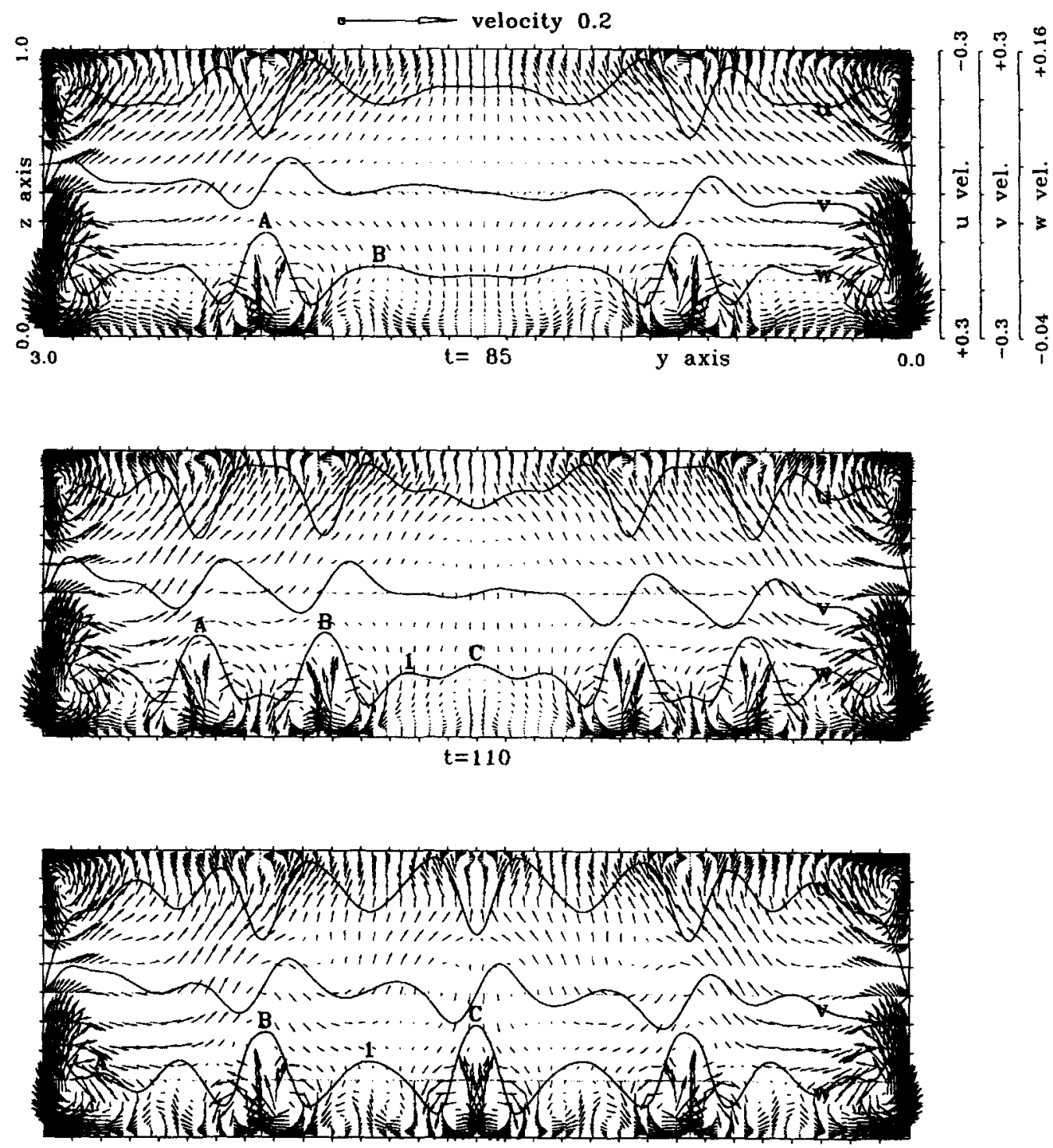

$t=138$

Figure 11. Evolution of TGL vortices at $x=0.525$ plane $(85 \leqslant t \leqslant 282)$. At $t=85$ the first pair of TGL vortices forms (marked by 'A'); at $t=110$ the second pair forms (marked by 'B'); at $t=138$ the third pair forms at the $y=1.5$ plane (marked by ' $C$ ') 
isosurface of $v=0$ and disrupts the flow pattern appreciably by means of a sliding-down vortex forming at $t=75$. The spatial location at which the waviness becomes visible in Figure 9 is exactly where the reflection of the pressure profile occurs. As seen in Figure 8, the contour surface of $v=0$ beings to emerge from the bottom surface at a location where two radial pressures of equal strength meet, as seen in Figure 9. This serves to indicate that the flow is prone to separation. Afterwards the separated flow extends in size and grows in strength.

This unsteady flow motion evolves continuously. At time $t=85$, vortices of the counter-rotating type form firstly at $y=2.25$ in Figure 8 . At the spanwise location $y=1.85$, developing TGL vortices accompany the well-developed pair of TGL vortices. Also, near the lid plane a less apparent pair of
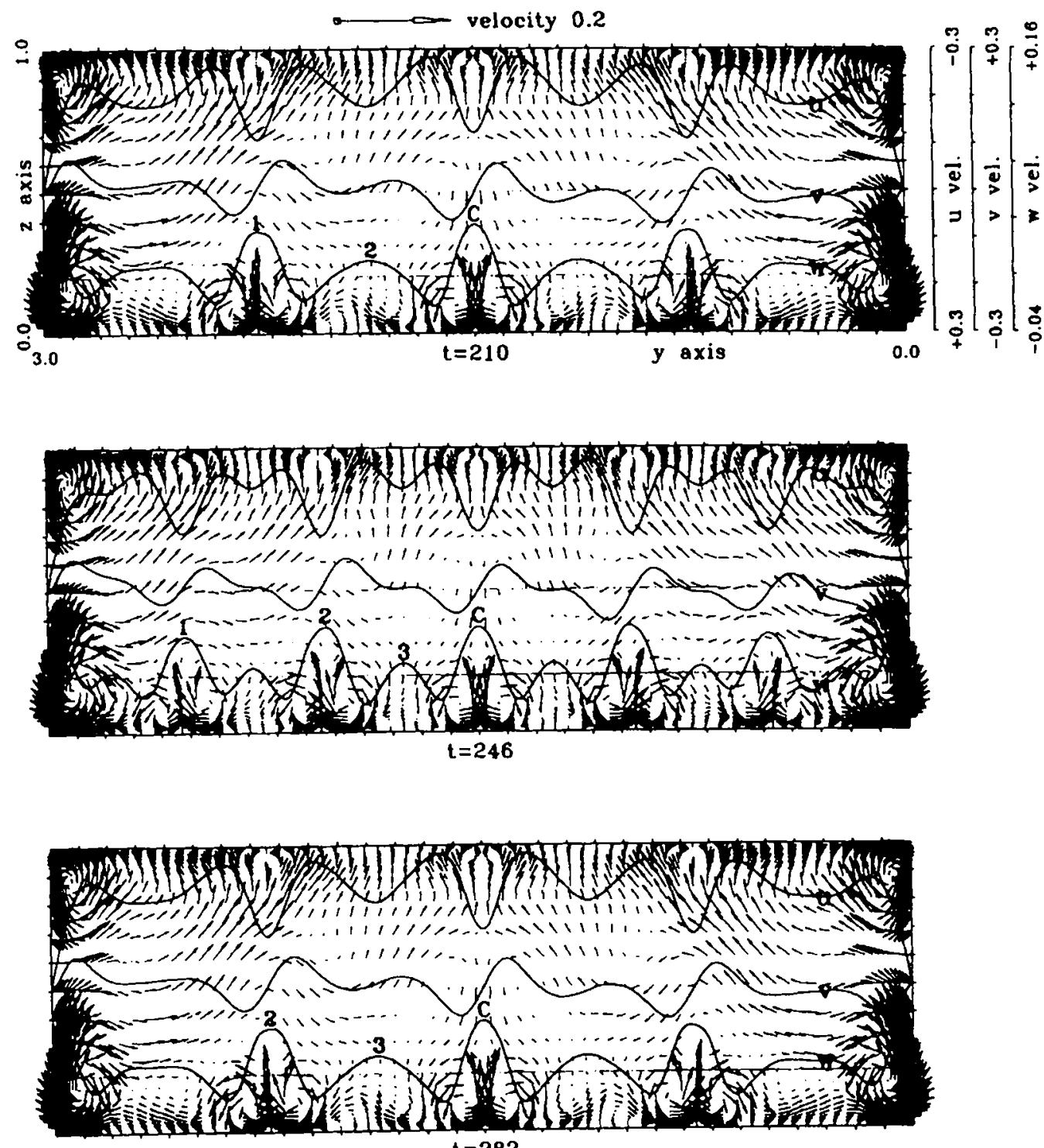

$t=282$

Figure 11. (continued) 
TGL vortices also forms at the same spanwise location. In fact, exclusive of the two end walls, TGL vortices can be observed along the solid surfaces of the cavity. By virtue of the exhibited global velocity distributions at time $t=85$, we interpret this oscillatory feature in Figure 7 as resulting from the flow instability. We further designate vortical instability of this sort as Taylor-Görtler vortices, mainly because they bear a strong resemblance to those observed in a concave boundary layer. These instabilities arise because of the centrifugal effects. Along the span, three wave-like velocity distributions, as shown in Figure 7, reveal that the crest of the w-profile coincides with the trough of $u$. This extreme location is exactly where the inflection point of the spanwise velocity $v$ is located. Noticeable is the cortex which seems to be almost immune to these Görtler instabilities.

On all accounts the onset of TGL vortices will considerably disnupt the general circulation and thus modify the pressure-driven secondary flow. Clearly visible from this figure is that the pressure field becomes protuberant between counter-rotating vortices, as shown in Figure 10. In the presence of counter-rotating vortices the spanwise spiral particle motion can no longer be classified solely as monotonic. Within the spanwise length of 0.12 in the range $2.5 \leqslant y \leqslant 2.25$ in Figure 10(b), back-andforth particle tracks, shown in Figure 4(c), come across the free shear part of a pair of TGL vortices. Apart from the TGL vortices present at $y=1.5$, for each pair of TGL vortices we classify the left vortex as being a wall-shear-induced vortex and the one at the right side as a free shear vortex in Figure $10(b)$. Between $t=65$ and 85 the protuberance in the $v=0$ tube, as shown in Figure 5, is indicative of the possible onset of TGL vortices. The question of whether TGL vortices are to be well developed depends on whether two vortices encountered can continuously retain energy from the primary core. The locations where the contour surface of $v=0$ apparently protrudes are the centres of the left and right vortices of TGL vortices, as shown in Figure 10. Here the onset of flow instability is attributable to the centrifugal/radial pressure imbalance force and the spanwise disturbance. Near the first pair of TGL vortices the spanwise pressure gradient, distorted radial pressure gradient and well-defined comer vortex jointly result in a new separation bubble, as shwon in Figure 8, from the bottom wall at time $t=80$. Later, at a time, say $t=85$, the size of this newly separated flow enlarges. This, together with the newly developed vortex at the isosurface of $v=0$, paves the way for the formation of another pair of TGL vortices.

In an attempt to illuminate the growth of TGL vortices, we also plotted the remaining transport sequences in Figure 11 for some selected times, from which some conclusions can be drawn. First, along the span, TGL vortices are not stationary but, rather, mobile. The two vortices which constitute a pair of unsymmetric TGL vortices vary little in size but slightly more in appearance. As compared with the centre of the free shear vortex in Figure 10, the centre of the wall-shear-induced rotation is located closer to the bottom wall. Second, the development of TGL vortices is not solely attributable to the damping mechanism. Third, the transport of TGL vortices manifests the complex dynamics present in the shear-driven cavity. Sustained by the spanwise pressure gradient, the small-scale trailing TGL vortices continue to be intensified. At $t=110$ the second pair of well-developed TGL vortices forms at approximately $y=2.05(0.95)$, whereas the first pair of TGL vortices has moved to $y=2.45(0.55)$. Until $\mathrm{t}=138$, one pair of symmetric TGL vortices emanates from $y=1.5$. At this moment the first pair of TGL vortices has been largely immersed in the comer vortices, while the second pair of welldeveloped TGL vortices has proceeded closer to the end wall at $y=2.25(0.75)$. In between the two sets of well-developed TGL vortices a pair of TGL vortices is developing. The sole pair of symmetric TGL vortices is referable to the wall shear vortices and will persist at $y=1.5$ in their ensuing development. At time $t=138$ the investigated mobile system reaches a periodic steady state. After than, TGL vortices emerge only at $y=1.73(1.27)$. In the subsequent periodic flow development from $t=210$ to 282 , three well-developed TGL vortices having a wavelength of 0.75 are equally distributed. At $t=246$, five sets of TGL vortices are predicted which are also uniforly distributed with a shorter 

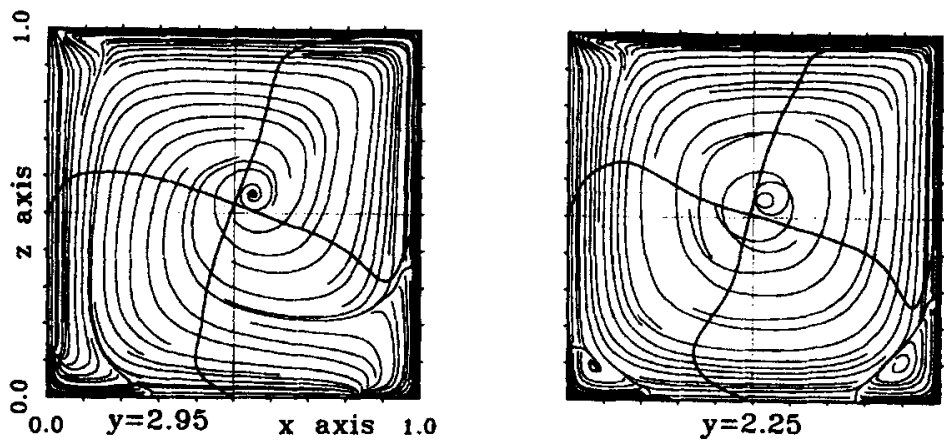

(a)
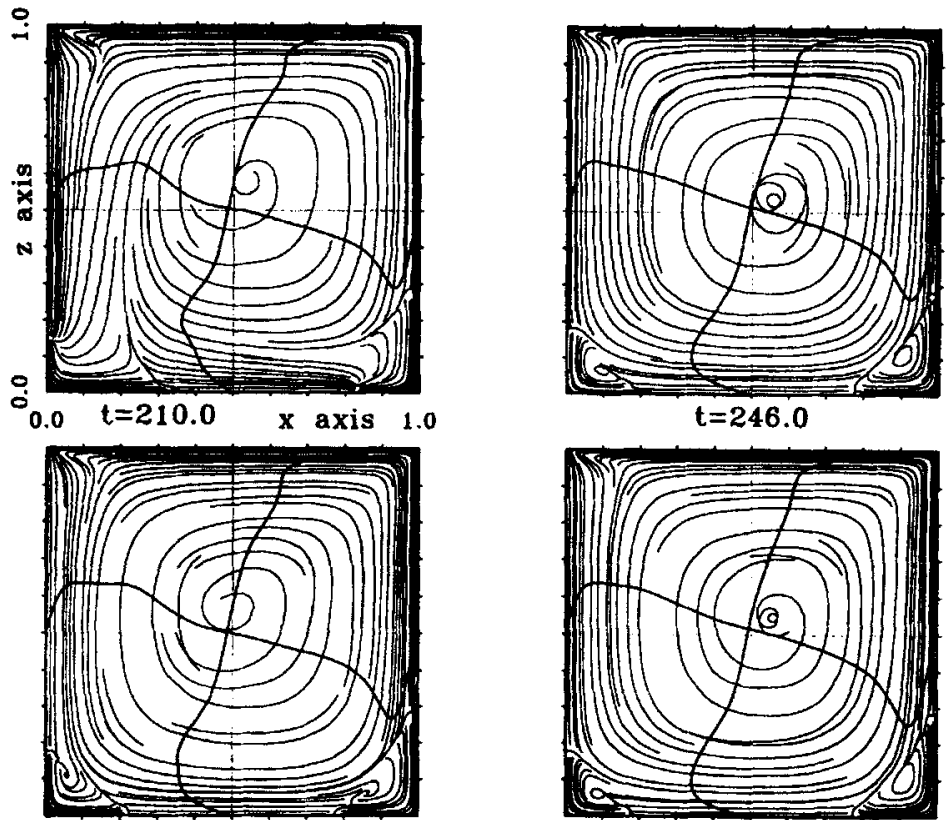

$t=246.0$

$t=222.0$

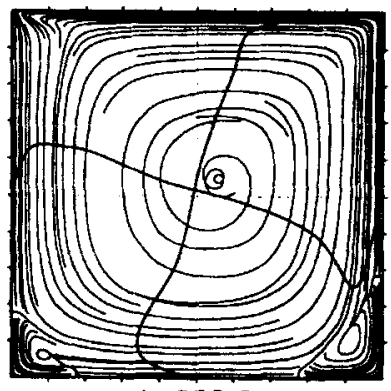

$t=258.0$
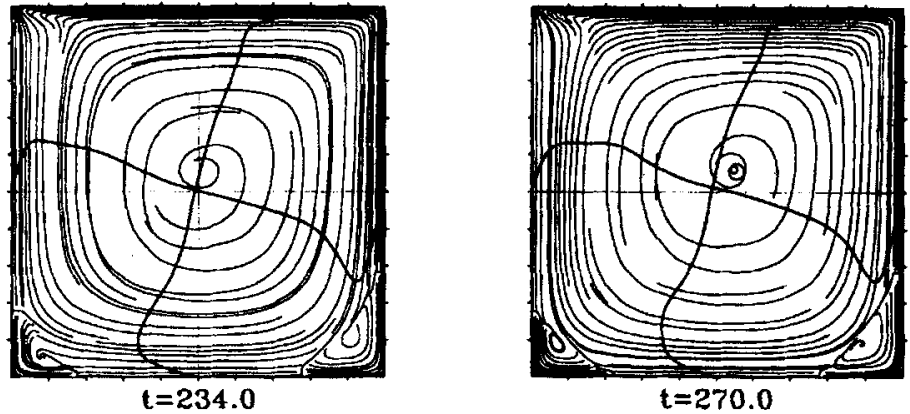

(b)

Figure 12. Influences of TGL vortices on primary flow plotted at $y$-planes: (a) at $y=2.95,2.25$ planes ( $t=25$ where no TGL is found); (b) at $y=2.25$ plane (within a period of TGL motion between $t=210$ and 282) 
wavelength of 0.5 . In support of the existence of flow periodicity, estimated to be 72 , we plot velocity vectors at $t=210,246$ and 282 in Figure 11.

It is worthwhile to point out the formation of TGL vortices does affect the primary flow structure. In order to show this, within a time period we have plotted solutions at the $y=2.25$ plane. For each time increment $\Delta t=12$, starting from 210 and terminating at 270 , computed solutions are given in Figure 12(b). At $t=210$ the primary flow structure is mostly affected by TGL vortices in that secondary eddies are disrupted as TGL vortices travel through. Like fluid particles near the end wall at the $y=2.95$ plane in Figure 12(a), fluid particles inside these eddies at $t=25$ are directly engulfed into the primary core of the flow. Afterwards, streamlines of a closed form appear again inside the DSE and USE. The primary flow pattern is less affected by TGL vortices at $t=246$, mainly because $y=2.25$ is where the middle of two well-developed TGL vortices is located. In these circumstances there exists a separation surface between the primary and the secondary eddy. Also, by comparing solutions computed at the plane $y=2.25$ in Figure 12(a), we can shed light on the influence of TGL vortices on the primary flow. As compared with the solutions at $t=25$ which have nothing to do with TGL vortices, the solution profiles at $t=246$ are maintained quite well at the downstream side. In the vicinity of the bottom and upstream sides the changes in velocity distributions are larger, especially at the upstream side. This implies that the upstream side is more sensitive to the TGL vortices.

\section{Trigger mechanism of TGL vortices}

Our concern here is to make an attempt to delineate what engenders the TGL instabilities. To begin with, we would like to distinguish corner vertices from TGL vortices because they resemble one another in appearance but not in character. As a fluid particle is forced to turn about a corner, the centrifugal force acting on this particle is balanced by an increasingly larger radial pressure. This yields a stable condition. Being destabilized by the concave streamlines, fluid flows are doomed to instability. This implies that the laminar instability should take possession of another distinct but influential component. Among other things, flow disturbances resulting from two vertical end walls give rise to the formation of TGL vortices. With these, pressure contours of a radial form emanate from a spatial location where the symmetry plane intersects the bottom wall. This perturbation propagates towards the end walls and consequently sets up a spanwise boundary layer on the bottom wall. In regions near the bottom wall, fluid particles moving spirally towards the two end walls encounter resistance at the stagnant corner. This sets up a flow environment as illustrated in Figure 10. The original pressure distribution that is mainly determined by the primary flow characteristics has been disrupted. The contours of pressure are thus modified over the span. According to the wavy pressure in Figure 10, the pressure in regions near the two ends has been increased, while it is decreased in between. This implies that a particle which is radially displaced by a centrifugal force fails to return to its original location because it encounters an insufficient pressure gradient force. Opposite to this behaviour, there remains a net centrifugal/radial pressure imbalance force in favour of the pressure gradient force at both ends. This brings about a counter-rotating flow motion, the axis of which is that of the longitudinal direction. Subsequent to this flow instability, unsymmetric counter-rotating vortices evolve periodically, because the locally intensified upward velocity vector bends to the end wall. This is due mostly to the pressure imbalance along the spanwise direction. The periodicity is estimated to be 7-8.

\section{CONCLUSIONS}

As a vehicle for providing physical insight into the rectangular shear-driven cavity in a non-uniform grid ssytem, we have used a QUICK-type scheme to discretize non-linear advective terms. The resulting coupled algebraic equations have been solved by employing a SIMPLE iterative algorithm. The underlying analysis code has been confirmed by conducting a non-trivial analytic test problem 
together with a spatial rate-of-convergence analysis. In the light of the rectified finite volume flow simulation, the following conclusions regarding the shear-driven cavity flow can be drawn.

1. Inside the rectangular cavity the vortical system is best depicted by spanwise sprialling flow motions. In the region of the primary core flow, particles spiral towards the symmetry plane and gradually return to the end wall region via the boundary layer. In illustration of the flow motion inside the primary vortex and secondary eddies, we plotted particle tracks.

2. Flow motion in a geometrically simple cavity is far from physically simple. The flow generated by a lid proceeds with a transient development. The flow structure established in the cavity is maintained by complex interactions among the large-scale primary recirculating flow, radial and spanwise pressure-driven force, secondary flow, centrifugal force, no-slip physical reality and the mobile TGL vortices observed in later transport sequences. In general the flow structure at the upstream side is less organized and less stable than the flow at the downstream side. Subsequent to the formation of the first set of TGL vortices at $y=2.25$, which are most clearly seen at the $x=0.525$ plane, the pressure-driven spanwise flow structure is affected by the unsymmetric counter-rotating vortices moving periodically towards the end wall.

3. Exploration of the mechanism leading to the flow instability has been the subject of the present numerical investigation. The developed TGL vortices proceed in a non-linear fashion. This is evident from the appearance of counter-rotating vortices. We interpret this class of laminar instability as being Taylor-Görtler instability, because this instability in the investigated viscous system is associated with concave streamlines. A sole centrifugal force, exerted radially between streamlines in possession of appreciable curvatures, fails to engender instability. Whether or not a flow system, stably maintained by the centrifugal force and the radial pressure gradient, yields instabilities depends on whether the existing spanwise perturbation is strong enough to break up such a balance. Here the spanwise pressure gradient resulting from the symmetry plane serves as the spanwise perturbation which may be amplified in the investigated viscous system containing concave streamlines. Of the predicted TGL vortices, the flow asymmetry is attributable to the wall-shear- and free-shear-induced mechanisms. As to the investigated Reynolds number $R e=1500$, the flow system can be characterized as symmetric. Within a half-cavity the first pair of unsymmetric TGL vortices forms at $y=2 \cdot 25$, followed by the second pair at $y=2.05$. Finally, a comparatively stationary pair of TGL vortices emerges at the symmetry plane.

\section{REFERENCES}

1. F. Pan and A. Acrivos, 'Steady flows in rectangular cavities', J. Fluid Mech., 28, 643-655 (1967).

2. V. Ya. Bogatyrev and A. V. Gorin, 'End effects in rectangular cavities', Fluids Mech.-Sov. Res., 7(4), 101-106 (1978).

3. J. R. Koseff, R. L. Street, P. M. Gresho, C. D. Upson, J. A. C. Humphrey and W. -M. To, 'A three-dimensional lid-driven cavity flow: experiment and simulation', Proc. 3rd Int. Conf. on Numerical Methods in Laminar and Turbulent Flow, Seattle, WA, August 1983, pp. 564-581.

4. J. R. Koseff and R. L. Street, 'Visualization studies of a shear driven three-dimensional recirculating flow', ASME J. Fluids Eng., 106, 21-29 (1984).

5. J. R. Koseff and R. L. Street, 'On the end wall effects in a lid-driven cavity flow', ASME J. Fluids Eng., 106, 385-389 (1984).

6. J. R. Koseff and R. L. Street, 'The lid-driven cavity flow: a synthesis of qualitative and quantitative observations', $A S M E J$. Fluids Eng., 106, 390-398 (1984).

7. A. K. Prasas and J. R. Koseff, 'Reynolds number and end-wall effects on a lid-driven cavity flow', Phys. Fluids $A, 1,208$ 218 (1989).

8. C. K. Aidun, N. G. Triantafillopoulos and J. D. Benson, 'Global stability of a lid-driven cavity with throughflow: flow visualization studies', Phys. Fluids A, 3, 2081-2091 (1991).

9. O. R. Burggraf, 'Analytical and numerical studies of the structure of steady separated flows', J. Fluid Mech., 24, 113-151 (1966). 
10. C. J. Freitas, R. L. Street, A. N. Findikakis and J. R. Koseff, 'Numerical simulation of three-dimensional flow in a cavity', Int. j. numer. methods fuids, 5, 561-575 (1985).

11. C. J. Freitas and R. L. Street, 'Non-linear transport phenomena in a complex recirculating flow: a numerical investigation', Int. j. numer. methods fuids, 8, 769-802 (1988).

12. H. C. Ku, R. S. Hirsch, T. D. Taylor and A. P. Rosenberg, 'A pseudospectral matrix element method for solution of threedimensional incompressible flows and its parallel implementation', J. Comput. Phys., 83, 260-291 (1989).

13. D. Tromeur-Dervout, L. Ta Phuoc and L. Mane, 'A 3D Navier-Stokes solver on distributed memory multiprocessor', K. W. Morton (ed.), Lecture Notes in Physics, Vol. 371, Springer-Verlag Berlin 1990 (Proc. Twelfth Int. Conf. on Numerical Methods in Fluid Dynamics, 1990, pp. 303-307).

14. C. Y. Perng and R. L. Street, 'Three-dimensional unsteady flow simulations: alternative strategies for a volume-averaged calculation', Int. j. numer: methods fluids, 9, 341-362 (1989).

15. Y. Kato, H. Kawai and T. Tanahashi, 'Numerical flow analysis in a cubic cavity by the GASMAC finite-element method', JSME Int. J. Ser. II, 33, 649-658 (1990).

16. A. B. Cortes and J. D. Miller, 'Numerical experiments with the lid driven cavity flow problem', Comput. Fluids, 23, 10051027 (1994).

17. Y. Zang, R. L. Street and J. R. Koseff, 'A non-staggered grid, fractional step method for time-dependent incompressible Navier-Stokes equations in curviliner coordinates', J. Comput. Phys., 114, 18-33 (1994).

18. N. Ramanan and G. M. Homsy, 'Linear stability of lid-driven cavity flow', Phys. Fluids, 6, 2690-2701 (1994).

19. O. A. Ladyzhenskaya, Mathematical Problems in the Dynamics of a Viscous Incompressible Flow, Gordon and Breach, New York, 1963.

20. S. Abdallah, 'Numerical solution for the incompressible Navier-Stokes equations in primitive variables using a nonstaggered grid II', J. Comput. Phys., 70, 193-202 (1987).

21. G. B. Deng, J. Piquet, P. Queutey and M. Visonneau, 'A new fully coupled solution of the Navier-Stokes equations', Int. $j$. numer. methods fluids, 19, 605-639 (1994).

22. C. M. Rhie and W. L. Chou, 'A numerical study of the turbulent flow past an isolated airfoil with trailing edge separation', AIAA J., 21, 1525-1532 (1983).

23. G. E. Schneider and M. J. Raw, 'Control-volume finite element method for heat transfer and fluid flow using collocated variables', Numer. Heat Transfer, 11, 363-400 (1987).

24. B. P. Leonard, 'A stable and accurate convective modelling procedure based on quadratic upstream interpolation', Comput. Methods Appl. Mech. Eng, 19, 59-98 (1979).

25. S. V. Patankar, Numerical Heat Transfer and Fluid Flow, Hemisphere, New York, 1980.

26. C. R. Ethier and D. A. Steinman, 'Exact fully 3D Navier-Stokes solutions for benchmarking', Int. $j$. numer. methods fuids, 19, 369-375 (1994).

27. H. Görtler, 'Über eine dreidimensionale Instabilität laminarer grenzschichten an konkaven Wänden', Nachr. Akad. Wiss. Göttingen Math. Phys. Kl. Neue Folge I, 2, 1-26 (1940).

28. J. Bennett, P. Hall and F. T. Smith, 'The strong nonlinear interaction of Tollmien-Schlichting waves and Taylor-Görtler vortices in curved channel flow', J. Fluid Mech., 223, 475-495 (1991). 